\title{
Glycyrrhetinic Acid-Poly(ethylene glycol)-glycyrrhetinic Acid Tri-Block Conjugates Based Self-Assembled Micelles for Hepatic Targeted Delivery of Poorly Water Soluble Drug
}

\author{
Fengbo Wu, ${ }^{1}$ Ting Xu, ${ }^{1}$ Chi Liu, ${ }^{1}$ Can Chen, ${ }^{2}$ Xiangrong Song, ${ }^{1}$ Yu Zheng, ${ }^{1}$ and Gu He \\ ${ }^{1}$ Department of Pharmacy and State Key Laboratory of Biotherapy, West China Hospital, Sichuan University, No. 37 Guoxue Alley, \\ Chengdu, Sichuan 610041, China \\ ${ }^{2}$ The First Affiliated Hospital of Chengdu Medical College, Chengdu, Sichuan 610500, China
}

Correspondence should be addressed to Gu He; heguscu@163.com

Received 24 June 2013; Accepted 29 July 2013

Academic Editors: D. Quintanar-Guerrero, A. Savaser, and L. A. Videla

Copyright (C) 2013 Fengbo Wu et al. This is an open access article distributed under the Creative Commons Attribution License, which permits unrestricted use, distribution, and reproduction in any medium, provided the original work is properly cited.

\begin{abstract}
The triblock $18 \beta$-glycyrrhetinic acid-poly(ethylene glycol)-18 $\beta$-glycyrrhetinic acid conjugates (GA-PEG-GA) based self-assembled micelles were synthesized and characterized by FTIR, NMR, transmission electron microscopy, and particle size analysis. The GAPEG-GA conjugates having the critical micelle concentration of $6 \times 10^{-5} \mathrm{M}$ were used to form nanosized micelles, with mean diameters of $159.21 \pm 2.2 \mathrm{~nm}$, and then paclitaxel (PTX) was incorporated into GA-PEG-GA micelles by self-assembly method. The physicochemical properties of the PTX loaded GA-PEG-GA micelles were evaluated including in vitro cellular uptake, cytotoxicity, drug release profile, and in vivo tissue distribution. The results demonstrate that the GA-PEG-GA micelles had low cytotoxicity and good ability of selectively delivering drug to hepatic cells in vitro and in vivo by the targeting moiety glycyrrhetinic acid. In conclusion, the GA-PEG-GA conjugates have potential medical applications for targeted delivery of poor soluble drug delivery.
\end{abstract}

\section{Introduction}

$18 \beta$-glycyrrhetinic acid (GA, 3 $\beta$-hydroxyl-11-oxo-olean-12ene-29-oic acid, 1 ) is one of the main active principles of the plant Glycyrrhizae radix, and GA is the hydrolysis active product of glycyrrhizic acid. GA has been used as medicine to treat allergic and hepatic diseases [1,2]. GA suppresses the tumor promoting effect of 12-O-tetradecanoylphorbol-13acetate (TPA) and 7,12-dimethylbenz[a]anthracene on skin tumor formation in mice [3] and has antitumor activities [4]. Recently, it has been shown that GA could induce apoptosis in human hepatoma, leukemia, and gastric cancer cells at high concentrations [5]. But, concomitantly, GA exhibited increased scarce stability and poor water solubility resulting in a decreased therapeutic index.

In the past few years, several research groups reported that the GA modified drug delivery systems bearing good hepatoma cellular targeted efficiency. Mao et al. found that the cellular uptake of liposomes modified with glycyrrhetinic acid by rat hepatocytes was 3.3-fold higher than that of unmodified ones [6]. Recently, Yuan and collaborators prepared chitosan nanoparticles, alginate nanoparticles, PLGA, and poly(ethylene glycol)-b-poly(gamma-benzyl Lglutamate) micelles modified with glycyrrhizic acid and confirmed that these modified nanoparticles or micelles preferentially accumulated in rat hepatocytes by a ligand-receptor interaction [7-17]. Tian et al. also reported a liver-targeted drug delivery carrier, composed of chitosan/poly(ethylene glycol)-glycyrrhetinic acid nanoparticles, prepared by an ionic gelation process, in which glycyrrhetinic acid acted as the targeting ligand [18]. In our previous study, we constructed glycyrrhetinic acid-modified stealth cationic liposomes loaded with pDNA and they were found to transfect human hepatocellular carcinoma cell line HepG2 with high efficiency [19]. Recently, the possibility of synthesizing a number of polyethylene glycol esters of GA and characterizing them to assess their chemical and enzymatic hydrolysis has been investigated [20-23]. But these polymerdrug conjugates have been designed as potential dermal prodrugs, and the short hydrophilic chain of oligoethylene 
esters cannot provide self-assembly properties. However, so far, there has been no consensus regarding which strategy provides the optimal strategy outcome. In our recent study, biodegradable GA-mPEG conjugates are synthesized by poly (ethylene glycol) monomethyl ether (mPEG). The mGAmPEG was synthesized through succinic anhydride chain which was used as a bridge for the attachment of GA with polyethylene glycol monomethyl. Meanwhile, GA-mPEG was synthesized without succinic anhydride chain. According to our results, there is no significant difference between the mGA-mPEG and the GA-mPEG micelles in their physicochemical properties, when they were formed under identical conditions [24]. And Tian et al. reported that there is no significant difference between the CTS/GA-PEG-GA NPs and the CTS/PEG-mGA NPs in their ability to target the liver, when they were formed under identical conditions. This indicated that the C3-hydroxyl group in GA has little influence on the targeting ability [25]. Although there had been demonstrated successes in deploying glycyrrhetinic acid as targeted group on hepatoma targeted delivery, however, many GA modified drug delivery systems were complicate, and some components were expensive or instable in room temperature.

In our most recent study, GA could become the hydrophobic fragment of GA-mPEG conjugates, and the conjugates could form self-assembly micelles bearing low cytotoxicity to HEK293 cell line [24]. Tian et al. reported the mixture of single and double modified GA-PEG conjugates that showed good ability to target the liver [25].

In the current study, the convenient, economic, and effective methods to prepare hepatoma targeting polymeric micelles, the surfaces of which are anchored with GA, have been successfully developed for drug delivery to the hepatoma cells. The aim of this paper was the synthesis of structurally uniformed glycyrrhetinic acid-poly(ethylene glycol)-glycyrrhetinic acid triblock conjugate (GA-PEG-GA) and to develop an intravenous formulation of paclitaxel utilizing nanomicellar technology to increase water solubility of PTX. GA-PEG-GA with controllable quality was easily synthesized in large amounts on an industrial scale and seemed to be a potent candidate as micellar carrier for PTX entrapment.

\section{Materials and Methods}

2.1. Materials. $18 \beta$-Glycyrrhetinic acid (GA, purity 98\%) was obtained from Fujie Chemical Co., Ltd. (Xian, China). Poly (ethylene glycol) $\left(\mathrm{MW}=2000, \mathrm{PEG}_{2000}\right)$, 1-(3-dimethylaminopropyl)-3-ethylcarbodiimide hydrochloride (EDCI), and dimethylaminopyridine (DMAP) were purchased from Sigma (St. Louis, MO, USA). Cholesterol (Chol) was obtained from Shanghai Bio Life Science \& Technology Co., Ltd. (Shanghai, China). All other chemicals were of analytical grade.

2.2. Synthesis of GA-PEG-GA Conjugates. The synthesis process of GA-PEG conjugates was displayed in Scheme 1. Synthesis of glycyrrhetinic acid methyl ester (GA-OMe, 3) and glycyrrhetinic acid methyl ester 3-O-hemisuccinate ester (GA-suc, 4) was carried out according to the literature [19, 24]. The synthesis process of GA-PEG-GA and GA-suc-PEGsuc-GA was briefly described as follows.

GA-PEG-GA: $18 \beta$-glycyrrhetinic acid $(4.0 \mathrm{~g}, 8.5 \mathrm{mmol})$, $\mathrm{PEG}_{2000}(6.8 \mathrm{~g}, 3.4 \mathrm{mmol})$, DMAP $(1.0 \mathrm{~g}, 8.19 \mathrm{mmol})$, and EDCI $(7.8 \mathrm{~g}, 40.9 \mathrm{mmol})$ were dissolved in $60 \mathrm{~mL}$ of dichloromethane. The solution was stirred viciously and refluxed at $39^{\circ} \mathrm{C}$ for $5 \mathrm{~h}$. After the reaction was completed, the solvent was removed by evaporation. The residue was dissolved in $20 \mathrm{~mL}$ of dichloromethane and washed twice with $1 \mathrm{~mol} / \mathrm{L} \mathrm{HCl}$ to remove DMAP. The combined organic layer was dried with anhydrous $\mathrm{Na}_{2} \mathrm{SO}_{4}$ and concentrated by rotary evaporation. The crude product was purified on a silica gel chromatography column, eluting with gradient ratio of dichloromethane/methanol from $100: 1$ to $10: 1$. The final product $\left(8.7 \mathrm{~g}\right.$, yellow tax) was obtained. The ${ }^{1} \mathrm{H}$ NMR spectrum of GA-PEG-GA was characterized using Bruker Avance 400 spectrometer $(400 \mathrm{MHz})$, and $\mathrm{CDCl}_{3}$ was used as the solvent. The samples were scanned from 400 to $4000 \mathrm{~cm}^{-1}$. ${ }^{1} \mathrm{H} \mathrm{NMR}\left(400 \mathrm{MHz}, \mathrm{CDCl}_{3}\right) \delta 5.77(\mathrm{~s}, 2 \mathrm{H}$, $12-\mathrm{H} * 2)$, 3.64-3.76 (m, 180H, $\left.\mathrm{PEG}_{2000}, \mathrm{CH}_{2} * 90\right), 2.80$ (dt, $J=13.6,4.5 \mathrm{~Hz}, 1 \mathrm{H}, 1 \beta-\mathrm{H}), 2.37$ (s, $2 \mathrm{H}, 9 \alpha-\mathrm{H} * 2), 1.37$ (s, $6 \mathrm{H}$, $\left.\mathrm{CH}_{3} * 2\right), 1.15$ (s, 6H, $\left.\mathrm{CH}_{3} * 2\right), 1.14$ (s, 6H, $\left.\mathrm{CH}_{3} * 2\right), 1.13$ $\left(\mathrm{s}, 6 \mathrm{H}, \mathrm{CH}_{3} * 2\right), 1.01\left(\mathrm{~s}, 6 \mathrm{H}, \mathrm{CH}_{3} * 2\right)$, and $0.81(\mathrm{~s}, 12 \mathrm{H}$, $\left.\mathrm{CH}_{3} * 4\right)$. The infrared spectra were also measured using an IR spectrometer (Nicolet 5DX FTIR). IR (film) $v\left(\mathrm{~cm}^{-1}\right)$ : 2886, 2696, 2239, 1727, 1658, 1146, and 1113.

GA-suc-PEG-suc-GA: the GA-suc-PEG-suc-GA conjugates (5) were prepared from dissolving $2.00 \mathrm{~g}(3.44 \mathrm{mmol})$ of 4 in $50 \mathrm{~mL}$ of dichloromethane. Then $0.42 \mathrm{~g}$ (3.44 mmol) of DMAP and $0.72 \mathrm{~g}(3.78 \mathrm{mmol})$ of EDCI and $3.54 \mathrm{~g}$ $(1.77 \mathrm{mmol}) \mathrm{PEG}_{2000}$ were added. The mixture was stirred for $12 \mathrm{~h}$ at room temperature, then DMAP and EDCI were washed off by $1 \mathrm{~mol} / \mathrm{L} \mathrm{HCl}$. The organic phases were collected and dried by $\mathrm{Na}_{2} \mathrm{SO}_{4}$ and the solvent was evaporated in vacuo. The products (5) were purified on a silica-gel column, eluting with a mixture of DCM-methanol $(60: 1)$. White solid (2.4 g, $27 \%)$ was obtained as ${ }^{1} \mathrm{H} \mathrm{NMR}\left(400 \mathrm{MHz}, \mathrm{CDCl}_{3}\right) \delta 5.77(\mathrm{~s}$, $2 \mathrm{H}, 12-\mathrm{H} * 2), 3.70$ (s, $\left.6 \mathrm{H},-\mathrm{OCH}_{3} * 2\right), 3.64-3.76(\mathrm{~m}, 180 \mathrm{H}$, $\left.\mathrm{PEG}_{2000}, \mathrm{CH}_{2} * 90\right), 2.80(\mathrm{dt}, J=13.6,4.5 \mathrm{~Hz}, 1 \mathrm{H}, 1 \beta-\mathrm{H}), 2.51-$ 2.73 (brs, $8 \mathrm{H}$, suc, $\mathrm{COCH}_{2} * 4$ ), 2.37 (s, $2 \mathrm{H}, 9 \alpha-\mathrm{H} * 2$ ), 1.37 (s, $\left.6 \mathrm{H}, \mathrm{CH}_{3} * 2\right), 1.15\left(\mathrm{~s}, 6 \mathrm{H}, \mathrm{CH}_{3} * 2\right), 1.14\left(\mathrm{~s}, 6 \mathrm{H}, \mathrm{CH}_{3} * 2\right), 1.13$ (s, $\left.6 \mathrm{H}, \mathrm{CH}_{3} * 2\right), 1.01\left(\mathrm{~s}, 6 \mathrm{H}, \mathrm{CH}_{3} * 2\right)$, and $0.81\left(\mathrm{~s}, 12 \mathrm{H}, \mathrm{CH}_{3} * 4\right)$; IR (film) $v\left(\mathrm{~cm}^{-1}\right): 2741,2696,1969,1732,1659$, and 1147.

\subsection{Determination of Critical Micelle Concentration (CMC).} The CMC of mPEG-Chol, GA-PEG-GA, and GA-suc-PEGsuc-GA was determined according to the literature [19]. Pyrene probe was used as a probe during CMC determination. The work was performed according to the characteristic of pyrene emission spectrum, a red shift of the band from $373 \mathrm{~nm}$ to $384 \mathrm{~nm}$ after being encapsulated into a micellar hydrophobic core. Appropriate amount of pyrene dissolved in acetone was added into clean flask and dried by nitrogen instrument. The dried mixtures were hydrated with $10 \mathrm{~mL}$ mPEG-Chol or GA-PEG-GA or GA-suc-PEG-suc-GA water solution at gradient concentrations. All the samples were 


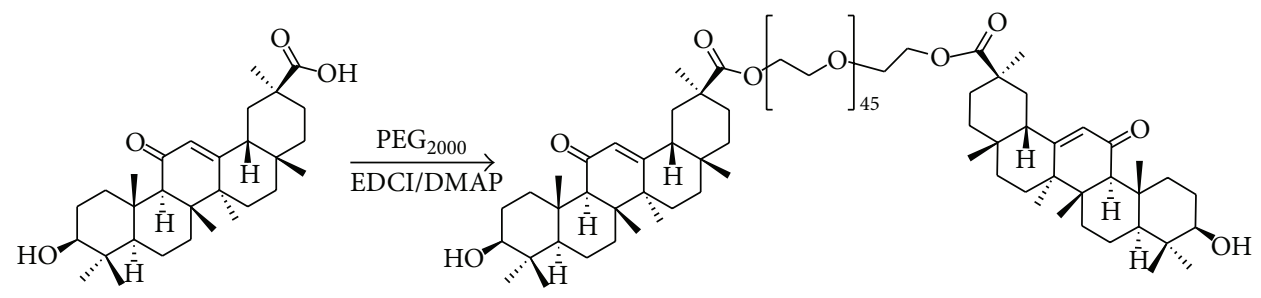

GA, 1

GA-PEG-GA, 2

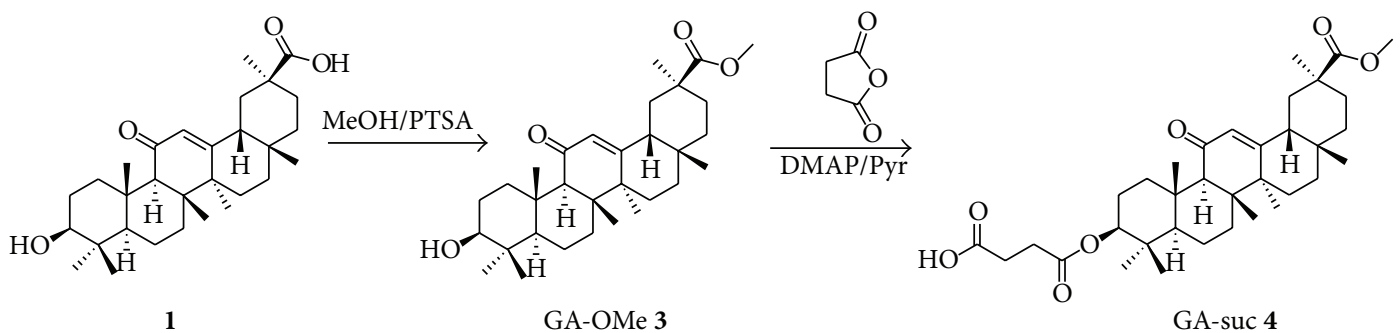

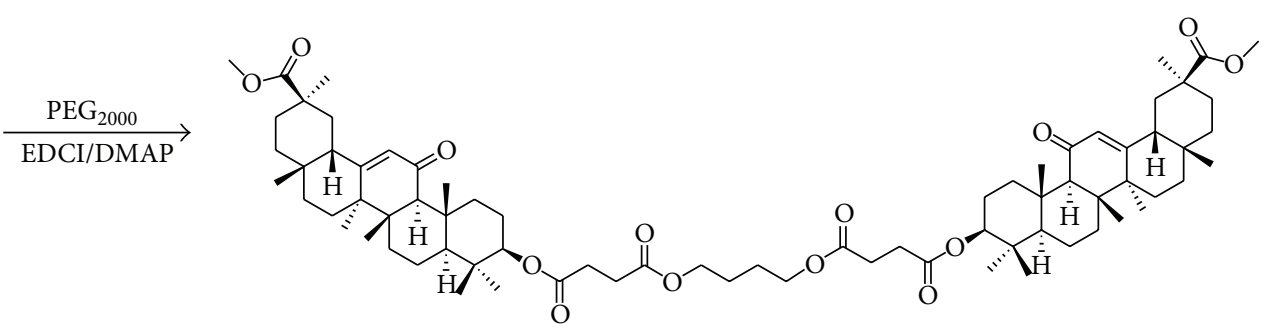

GA-suc-PEG-suc-GA 5

Scheme 1: Synthesis of GA-PEG-GA and GA-suc-PEG-suc-GA conjugates.

detected on fluorescence spectrophotometer with excitation wavelength at $335 \mathrm{~nm}$ and emission wavelengths at $373 \mathrm{~nm}$ (I1) and $384 \mathrm{~nm}$ (I3). The CMC value was taken from the intersection of the tangent to the curve at the inflection with the horizontal tangent through the points at low concentrations.

2.4. Micelle Formation and Drug Loading. GA-PEG-GA micelles (GA-M) were prepared using thin film hydration method. To prepare paclitaxel loaded GA-PEG-GA micelles (GA-M-PTX), $1.0 \mathrm{mg}$ of paclitaxel and $5 \mathrm{mg}$ GA-PEG-GA were dissolved in $5 \mathrm{~mL}$ mixed solvent of acetone and chloroform $(\mathrm{v} / \mathrm{v}=1: 4)$ at room temperature. The solvents were evaporated under vacuum at $37^{\circ} \mathrm{C}$ for $30 \mathrm{~min}$ to form a dry drug-containing lipid film. The formed dried lipid film was hydrated with $20 \mathrm{~mL}$ Mili-Q water at $40^{\circ} \mathrm{C}$ and then sonicated in water bath for $30 \mathrm{~min}$. The micelle was centrifuged at $1500 \mathrm{rpm}$ for $10 \mathrm{~min}$ and extruded through $220 \mathrm{~nm}$ filter to remove unloaded drugs. The final amount of capsulated paclitaxel was measured by high-performance liquid chromatography (HPLC) analysis.

The coumarin loaded micelles (GA-M-Cou) were prepared by similar method of GA-M-PTX, and the ratio of coumarin to GA-PEG-GA was 1 to 200 (w/w).

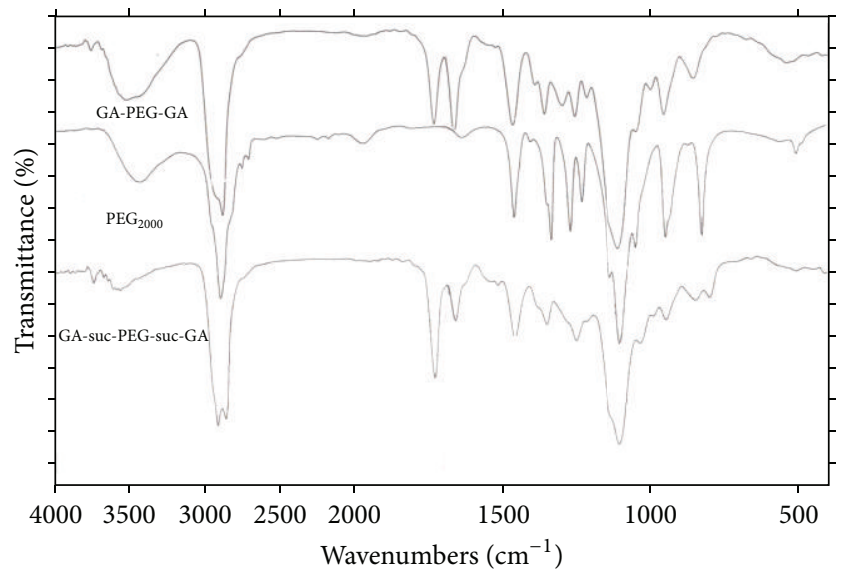

FIgURE 1: FT-IR spectra of PEG $_{2000}$, GA-PEG-GA, and GA-sucPEG-suc-GA.

mPEG-Chol micelle (Chol-M-Cou) was prepared by dropping the solution of $\mathrm{MPEG}-\mathrm{Chol}$ and coumarin in DCM $(2 \mathrm{~mL})$ into $20 \mathrm{~mL}$ Mili-Q water and stirred overnight. The micelle solution was evaporated for $30 \mathrm{~min}$ to remove organic solvents. The amount of mPEG-Chol and coumarin used was the same to GA-M-Cou. 


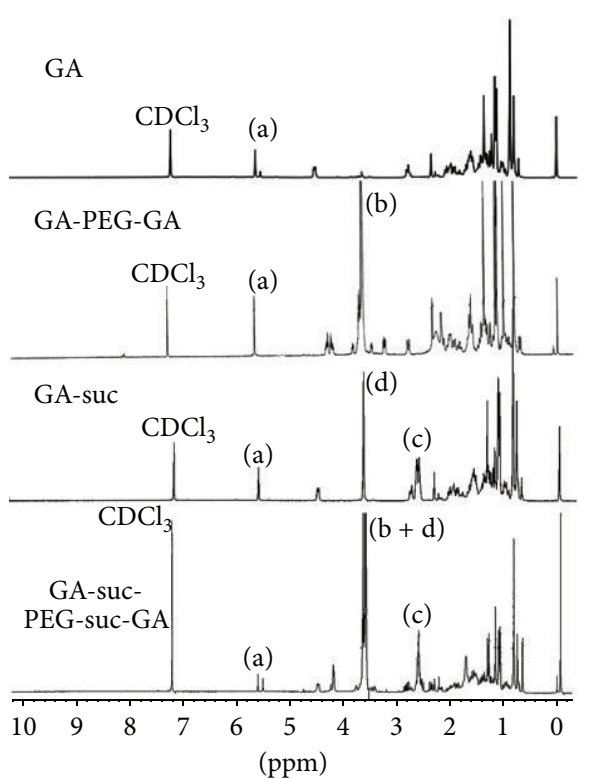

Figure 2: ${ }^{1} \mathrm{H}$ NMR spectra of GA and GA-PEG-GA and GA-sucPEG-suc-GA.

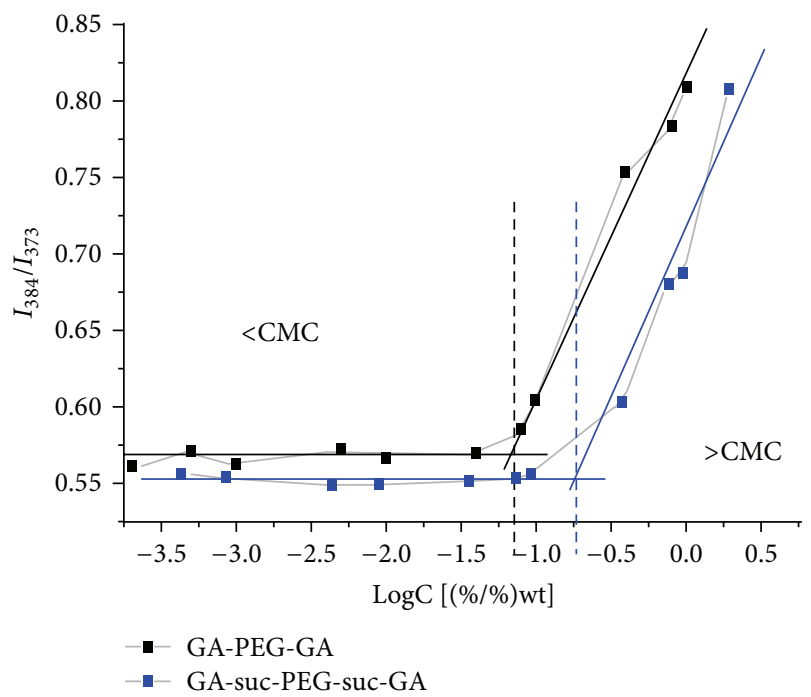

FIgUre 3: The CMC (critical micelle concentration) of GA-PEG-GA and GA-suc-PEG-suc-GA.

2.5. Size and Zeta-Potential Determination. Particle size and zeta potential of the micelle were determined by dynamic light scattering (DLS) with a Zetasizer Nano ZS-90 instrument (Malvern Instruments, Malvern, UK). Refractive index was 1.330 and temperature was kept at $25^{\circ} \mathrm{C}$ during measuring process. The micelle suspension was kept at $25^{\circ} \mathrm{C}$ during measuring process. All tests were run 3 times and took mean values.

2.6. Transmission Electron Microscopy (TEM). The morphology of PTX-loaded micelles was observed by TEM (H-600, Hitachi, Japan). Before analysis, the samples were diluted 1:5 and negatively stained with $2 \%(\mathrm{w} / \mathrm{v})$ phosphotungstic acid for $30 \mathrm{~s}$ and then placed on copper grids precoated with a thin film of polyvinyl formaldehyde for observation.

2.7. In Vitro Drug Release. The release profile of paclitaxel from micelles was investigated using a dialysis method. The test was performed on a thermostatic shaker. Briefly, $4 \mathrm{~mL}$ GA-M-PTX solution was placed in a dialysis bag (molecular weight cutoff $=1.0 \mathrm{kDa}$ ), which was suspended in $150 \mathrm{~mL}$ PBS ( $\mathrm{pH} 7.40 .1 \mathrm{M}$ ) with $0.2 \%$ Tween- 80 at $37^{\circ} \mathrm{C}$ with shaking at a speed of $100 \mathrm{r} / \mathrm{min} .1 \mathrm{~mL}$ aliquots were withdrawn and replaced with the equal volume of fresh medium at appropriate time intervals. HPLC was performed to determine the concentration of PTX in recovered release medium.

2.8. In Vitro Cytotoxicity Assay. Three kinds of tumor cell (Hela and HepG2) were chosen for the treatment experiment and cultured in DMEM medium containing 10\% FBS. Cellular cytotoxicity of blank GA-PEG-GA micelles, paclitaxel loaded GA-PEG-GA micelles (GA-M-PTX), and paclitaxel loaded mPEG-Chol micelles (Chol-M-PTX) were evaluated by MTT (3-[4,5-dimethylthiazol-2-yl]-2,5-diphenyl tetrazolium bromide) assay. Cells were seeded in 96-well plates at a density of $3 \times 10^{3}$ cells per well in $100 \mathrm{uL}$ DMEM. After $24 \mathrm{~h}$ of incubation at $37^{\circ} \mathrm{C}$ with $5 \% \mathrm{CO}_{2}$, cells were treated with different concentrations of blank micelle, CholM-PTX, and GA-M-PTX solution (each well with a ratio of medium component to nonmedium component equivalent to $9: 1)$, respectively. 48 hours later, $20 \mathrm{uL} \mathrm{MTT}(5 \mathrm{mg} / \mathrm{mL}$, dissolved in physiologic saline) was added to each well and incubated for another $4 \mathrm{~h}$. Then the incubated medium was removed, added $150 \mathrm{uL}$ DMSO to each well, and gently shook for $10 \mathrm{~min}$ at room temperature. Absorbance was measured at $570 \mathrm{~nm}$ using a Spectramax M5 Microtiter Plate Luminometer (Molecular Devices, USA).

2.9. Cellular Uptake Experiment. To evaluate the ability of GA-PEG-GA micelles binding to heptoma cells (hepG2), a micelle made of mPEG-Chol was used as a negative control. The cellular uptake tests of GA-PEG-GA micelle and mPEG-Chol micelles were performed by microscope and flow cytometric assay. HepG2 cells were seeded in 6-well plates at a density of $2 \times 10^{5}$ cells per well and cultured for $24 \mathrm{~h}$ in $1.8 \mathrm{~mL}$ DMED supplemented with $10 \%$ fetal bovine serum (FBS), at $37^{\circ} \mathrm{C}$ in $5 \% \mathrm{CO}_{2} .200 \mathrm{uL}$ of GA-M-Cou and Chol-MCou solution loading the equal quantity ( $0.1 \mathrm{ug}$ per well) of coumarin was added to predesigned wells. After incubation for $3 \mathrm{~h}$ and $6 \mathrm{~h}$, respectively, medium was removed, and cells were washed with physiological saline 3 times. The fluorescence of coumarin that entered into the tumor cells was observed through a fluorescence and light microscope (Olympus IX71, Olympus, Japan). Then cells were lysed with Trypsin and collected. Fluorescence intensity of coumarin was measured by flow cytometry.

2.10. Tissue Distribution of Coumarin Loaded GA-PEG-GA Micelles. The rats received $1 \mathrm{mg} / \mathrm{kg}$ of coumarin loaded GAPEG-GA micelles and equivalent amount of the mixture of free coumarin and blank GA-PEG-GA micelles in normal 


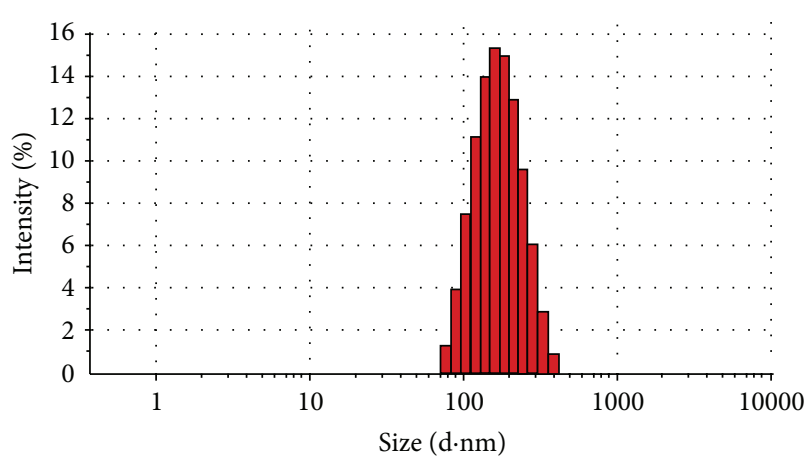

(a)

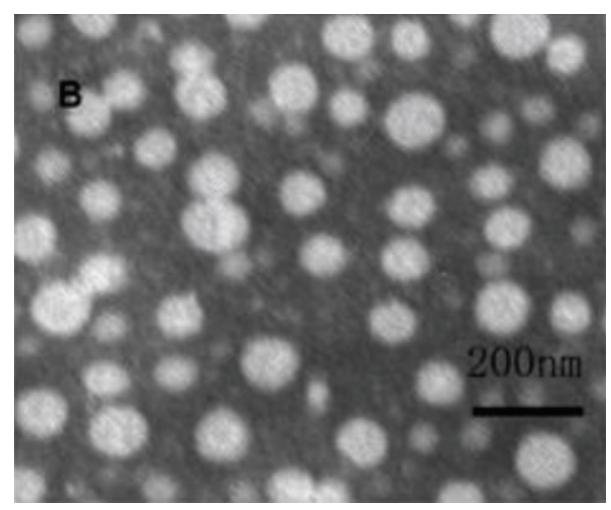

(b)

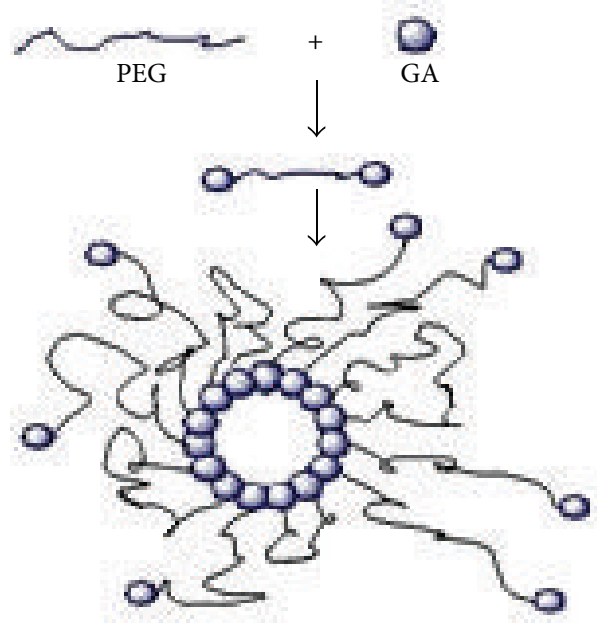

(c)

FIgURE 4: Preparation and characterization of the paclitaxel loaded GA-PEG-GA micelles. (a) Size distribution of the paclitaxel loaded GAPEG $_{2000}$-GA micelles (GA-M-PTX); (b) TEM image of GA-M-PTX (size of the scale bar: $200 \mathrm{~nm}$ ); (c) diagram of the preparation of GAPEG-GA micelles.

saline intravenously, respectively. The rats were sacrificed at predetermined time points and intestines were immediately dissected to expose the main organs before imaging. After intravenous injection, fluorescence imaging was recorded after 4 hours and the real-time images were performed in macroimaging system LT-9 equipped with illumatool dual light system LT-99D2 (Lightools Research, Encinitas, CA, USA).

\section{Results and Discussion}

3.1. Synthesis and Characterization of GA-PEG-GA Conjugates. The GA-PEG-GA and GA-suc-Peg-suc-GA conjugates were successfully prepared by using the synthetic route as shown in Scheme 1. The synthesis process of methoxylpoly(ethylene glycol)-cholesterol conjugate (mPEG-Chol) was reported in our previous studies and other literature studies [26-29]. The method of choice for coupling bioactive components to the PEG backbone is mainly by esterification. This type of esterification can be divided into two main approaches: (a) activation of the hydroxy end group through transformation into a good leaving group and subsequent attack by the carboxylate component, such as the formation of PEG-isourea and PEG-tosylate and (b) activation of the carboxy component and subsequent attack by PEG hydroxy end groups, such as the direct coupling by carbodiimide. Particularly, it was demonstrated that DMAP catalyzed attachment of carbodiimide-activated glycyrrhetinic acid proceeds good yield with PEG under very mild conditions. Therefore, we employed this EDCI/DMAP method in the synthesis of both PEG-amino acid derivatives and the final glycyrrhetinic acid-PEG-glycyrrhetinic acid conjugates.

The FT-IR spectra of PEG, GA-PEG-GA, and GA-sucPEG-suc-GA were shown in Figure 1. The broad band at around $3480 \mathrm{~cm}^{-1}$ attributed to the inter- and intramolecular hydrogen bonding of $-\mathrm{OH}$ stretching vibration of $3-\mathrm{OH}$ of glycyrrhetinic acid in GA-PEG-GA, because the corresponding hydroxyl groups in $\mathrm{PEG}_{2000}$ were primary hydroxyl groups, and the band had moved to around $3390 \mathrm{~cm}^{-1}$. The strong peaks around $2880 \mathrm{~cm}^{-1}$ were assigned to the $-\mathrm{CH}_{2}-$ which was brought by PEG. The sharp peak at $1112 \mathrm{~cm}^{-1}$ was assigned to the $\mathrm{C}-\mathrm{O}$ group which was brought by the PEG. Another small peak at $1730 \mathrm{~cm}^{-1}$ belongs to the carbonyl group of ester linkage between glycyrrhetinic acid 


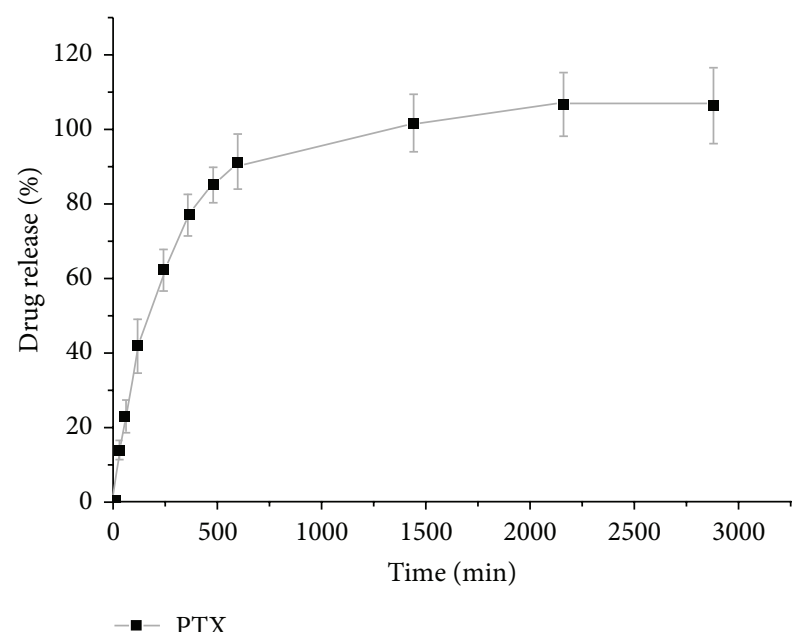

FIGURE 5: In vitro release profiles of PTX from GA-PEG-GA micelles in PBS at $\mathrm{pH} 7.4$.

and PEG. Other prominent peaks at $1340 \mathrm{~cm}^{-1}$ and $1460 \mathrm{~cm}^{-1}$ were assigned to the asymmetrical and symmetrical bending vibrations of methyl, methylene groups which were the introduction of long PEG chain.

Figure 2 showed the ${ }^{1} \mathrm{H}$ NMR spectra of GA, GA-PEG$\mathrm{GA}$ and GA-suc-PEG-suc-GA in $\mathrm{CDCl}_{3}$. The single peak at $\delta 5.69$ (a) was attributed to the protons of olefinic bond ($(\mathrm{C}=\mathrm{O})-\mathrm{CH}=\mathrm{C}-)$ in $\mathrm{GA}$. The peaks at $\delta 3.52-3.76((\mathrm{~b})$ and (d)) were attributed to the protons from the glycol unit ($\mathrm{CH}_{2}-\mathrm{CH}_{2}-\mathrm{O}-$ ) in PEG chain and the protons from methyl ester. The peaks at $\delta 2.58-2.66$ (c) came from the protons of succinate linkage $\left(-(\mathrm{C}=\mathrm{O})-\mathrm{CH}_{2}-\mathrm{CH}_{2}-(\mathrm{C}=\mathrm{O})-\right)$. Thus the GA-PEG-GA conjugates have been successfully synthesized.

3.2. Determination of Critical Micelle Concentration (CMC). The CMC is an important characteristic for amphiphilic copolymer, demonstrating the self-aggregation of copolymer. To determine the CMC of GA-PEG-GA and GA-suc-PEGsuc-GA, the transition of emission wavelength of pyrene between being free in water and entrapped into micelle was detected. Figure 3 was made with $I_{3} / I_{1}$ as the $Y$-axis and $\log \mathrm{C}$ as the $X$-axis, and an inflection point was shown by value of $\log C$. The CMC value of GA-PEG-GA and GA-sucPEG-suc-GA was shown to be as low as $6.0 \times 10^{-5} \mathrm{M}$ and $3.1 \times 10^{-4} \mathrm{M}$, respectively, which was similar to the CMC values of $\mathrm{PEG}_{2000}-\mathrm{PE}$ and $\mathrm{mPEG}_{2300}-\mathrm{DSPE}$ determined by the method of pyrene fluorescence probe and $\mathrm{mPEG}_{2200^{-}}$ cholesterol determined by the method of surface tension [2325]. These results indicated that the GA-PEG-GA micelles had lower CMC and higher stability and thereby might retain the integrity even upon strong dilution during systemic circulation. Therefore, the GA-PEG-GA micelles were chosen to perform the subsequent experiments.

3.3. Preparation and Characterization of Blank and Drug Loaded GA-PEG-GA Micelles. The PTX loaded GA-PEG-GA micelles were found to have a mean diameter of $159.21 \pm 2.22$ (mean $\pm \mathrm{SD} ; n=3$ ), with a distribution from 78.8 to $396.1 \mathrm{~nm}$ $(\mathrm{PDI}=0.123)$ (Figure 4(a)), which, compared with blank micelle, showed a slight increase in micelle size. The surface charges of GA-M-PTX were $-18.53 \pm 0.80 \mathrm{mV}$ (mean $\pm \mathrm{SD}$; $n=3$ ) in zeta potential measurement, which demonstrated the high stability of GA-M-PTX in water. Polydispersity in the micelle diameter can be attributed to a molecular weight distribution. The micelle size represented in the TEM images appeared to be smaller than that detected by DLS (Figure 4(b)). The scheme for the preparation of the GAPEG-GA micelles was displayed in Figure 4(c). Hydrodynamic radii of particles determined using DLS are typically found to be significantly larger than those determined by TEM. This is seen because the particle radius is based on the intensity of scattered light so the size is skewed toward larger particles. One additional possibility for this difference is the inability to view the corona of the micelle. One would see the corona if the micelles were very densely packed.

Encapsulating the insoluble drug is an important function of micelles. To evaluate the characteristic of micelle loading PTX, $1 \mathrm{~mL}$ methanol was added into $1 \mathrm{~mL}$ GA-M-PTX solution and sonicated for $30 \mathrm{~min}$. HPLC was performed, and the quantity of PTX was determined based on a linear standard curve obtained at a concentration range of $0.002-$ $0.01 \mathrm{mg} / \mathrm{mL}$.

The gradient ratio of PTX/GA-PEG-GA used in formulation was determined. When the ratio of PTX to GA-PEG-GA was $1: 5$, the loading efficiency and loading content reached an optimal value, which were equivalent to $59.8 \% \pm 1.4 \%$ $($ mean $\pm S D)$ and $13.0 \% \pm 0.3 \%($ mean $\pm S D)$.

3.4. In Vitro Release Profile of Paclitaxel from GA-PEG-GA Micelle. The in vitro release profile of paclitaxel from GAPEG-GA micelles (GA-M-PTX) was studied at $37^{\circ} \mathrm{C}$ and $\mathrm{pH}$ 7.0. Data suggests that paclitaxel can be well encapsulated in GA-PEG-GA micelles and released in an extended period. As shown in Figure 5, after $10 \mathrm{~h}, 91.49 \%$ of total paclitaxel was released from GA-M-PTX, followed by complete release after $36 \mathrm{~h}$.

3.5. In Vitro Cytotoxicity Assay. To evaluate the cytotoxicity of PTX loaded GA-PEG-GA micelles, HpeG2 and Hela cell lines were chosen for MTT analysis. As shown in Figure 6, PTX loaded GA-PEG-GA micelles were comparable to blank GA-PEG-GA micelles and PTX loaded mPEG-Chol micelles in antitumor activity in both cancer cell lines (HepG2 and Hela), and there was no significant difference of cytotoxicity observed between GA-M-PTX and Chol-M-PTX on Hela cell line, the $\mathrm{IC}_{50}$ values were $0.22 \mathrm{ug} / \mathrm{mL}$ for Chol-MPTX and $0.20 \mathrm{ug} / \mathrm{mL}$ for GA-M-PTX, respectively $(P<$ 0.01). As shown in Figure 6(b), GA-M-PTX showed slightly higher cytotoxicity than Chol-M-PTX. The mean concentrations of paclitaxel that caused $50 \%$ cell inhibition $\left(\mathrm{IC}_{50}\right)$ of GA-M-PTX were decreased to $0.32 \mathrm{ug} / \mathrm{mL}$ compared with $0.54 \mathrm{ug} / \mathrm{mL}$ of Chol-M-PTX, respectively. The drugfree GA-M did not show obviously cytotoxicity to the two cells. 


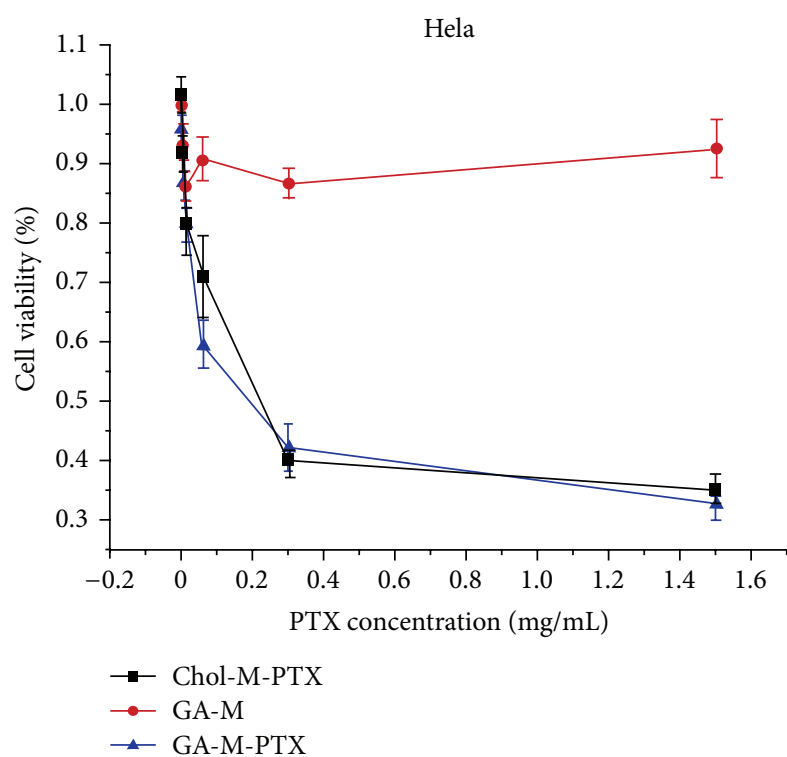

(a)

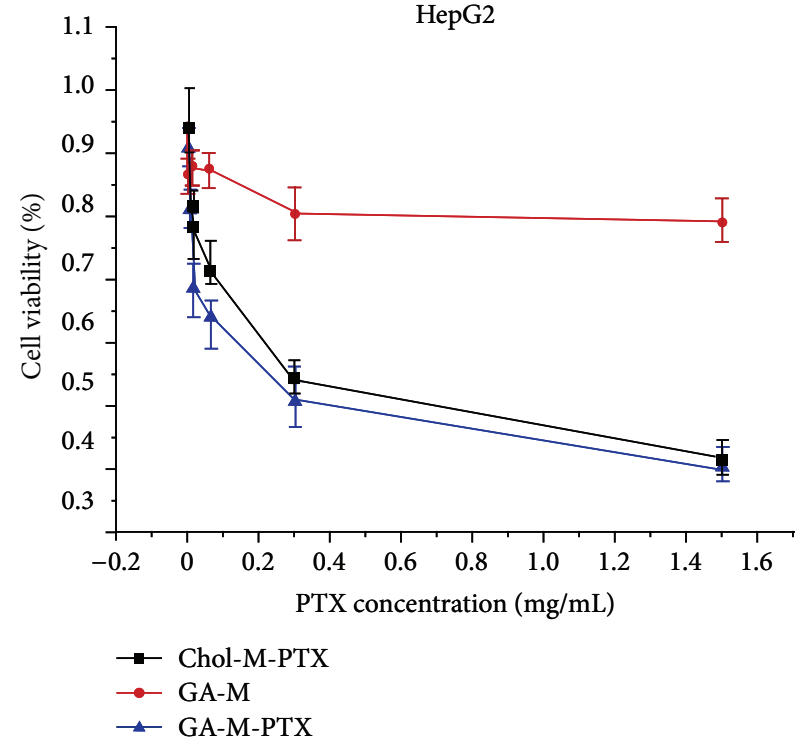

(b)

Figure 6: Cytotoxicity of Chol-M-PTX ( $\bullet$ ), blank micelles $(\bullet)$, and GA-M-PTX ( $\mathbf{\Delta})$ on Hela (a) and HepG2 (b) cell lines. The percentage of viable cells was quantified using the MTT method. Mean values and 95\% confidence intervals derived from three independent experiments are shown. $P<0.05$.

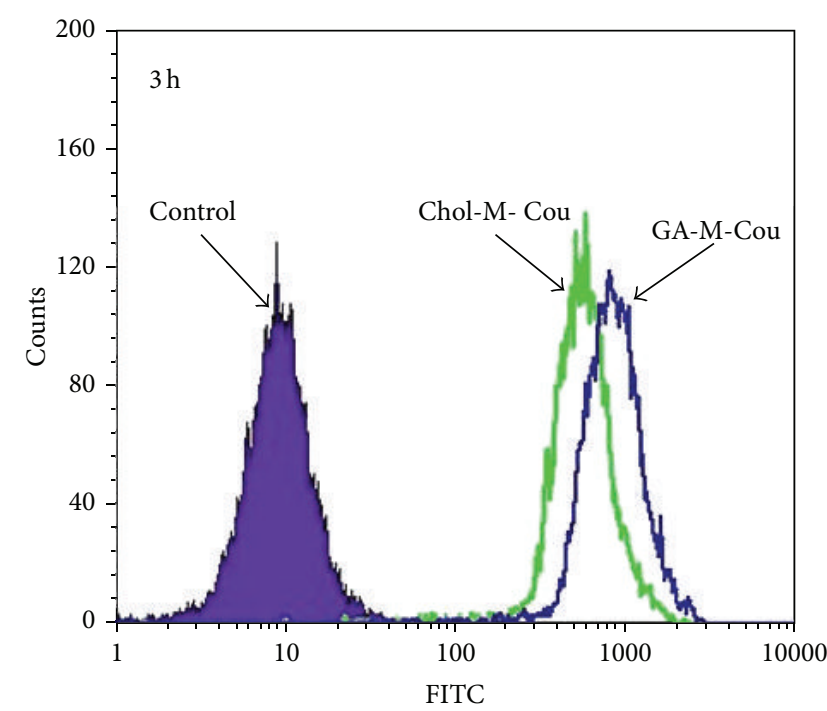

(a)

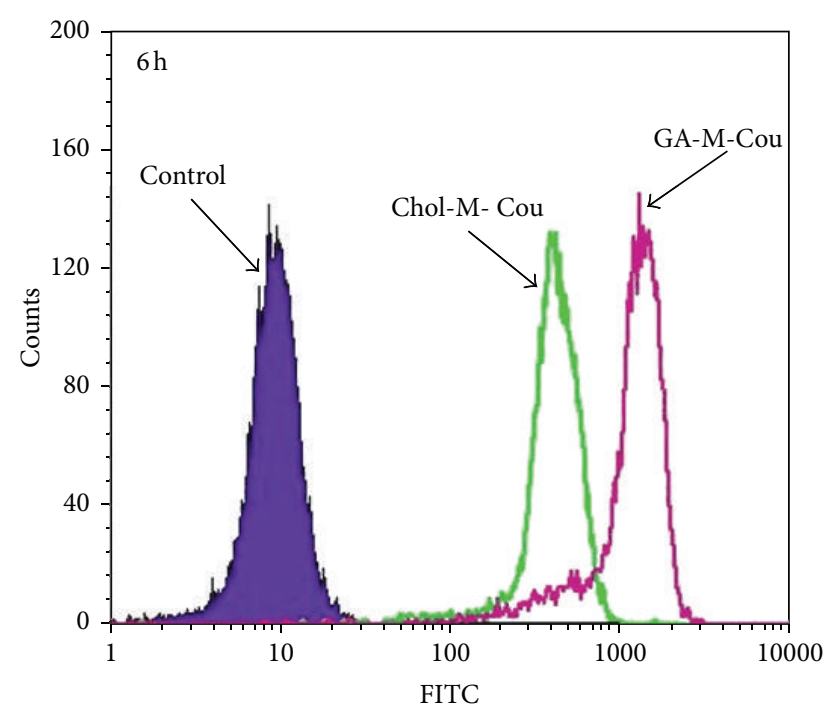

(b)

FIgURE 7: Flow cytometry profiles of hepG2 cell line after 3h (a) and 6h (b) incubation with control, Chol-M-Cou, and GA-M-Cou.

3.6. Cellular Uptake Experiment. To evaluate the effect of glycyrrhetinic acid block in micelle binding to hepatoma cells, mPEG-Chol micelle (Chol-M) was made as a negative control. HepG2 cells were treated with both micelles (GA-M and Chol-M) encapsulating with coumarin. After incubation for specific time, cells were washed and identified using a fluorescence microscopy. Then cells were collected, and the intensity of coumarin was measured by flow cytometric assay.

The flow cytometry data (Figure 7) shows the distribution of the intensity of coumarin encapsulated into
HepG2 cells after with incubated with GA-M-Cou, Chol-MCou, or empty GA-PEG-GA micelles (control). The mean fluorescence intensity of coumarin uptaken by cells after $3 \mathrm{~h}$ and $6 \mathrm{~h}$ incubation clearly showed that the difference of fluorescence intensity between GA-M-Cou and Chol$\mathrm{M}$-Cou for $6 \mathrm{~h}$ incubation become much greater compared with the ones for $3 \mathrm{~h}$ incubation. The percentage of positive cells containing coumarin after $6 \mathrm{~h}$ incubated with GA-M-cou was significantly increased by 5.1fold in comparison to that incubated with Chol-M-Cou 


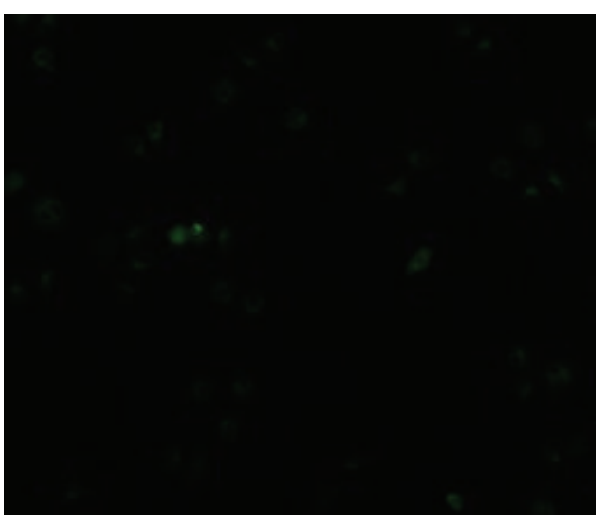

(a)

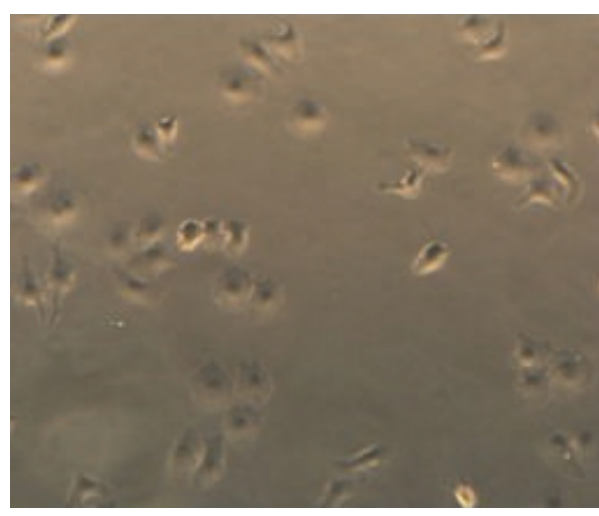

(c)

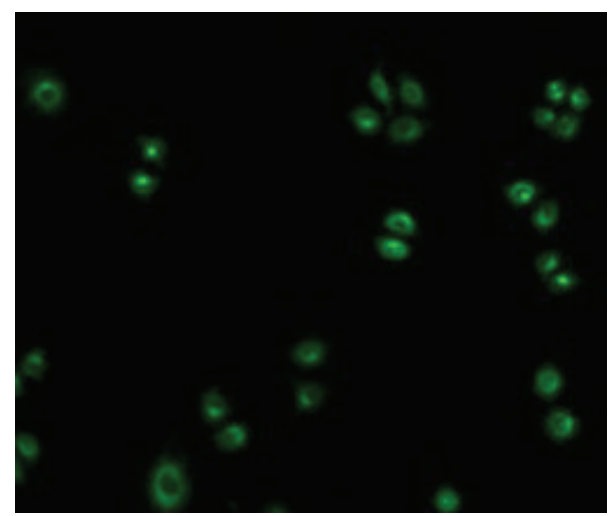

(b)

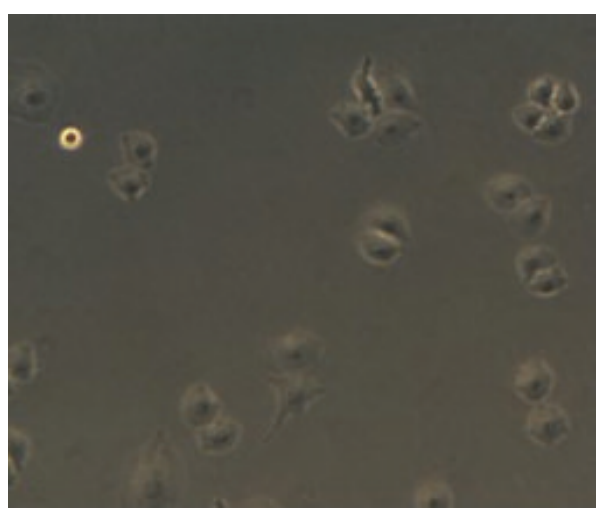

(d)

FIGURE 8: The fluorescence microscopy images and white light microscopy images of HepG2 cells after $6 \mathrm{~h}$ incubation with different coumarin loaded micelles: the Chol-M-Cou ((a) and (b)); the GA-M-Cou ((c) and (d)).

with the same time (Figure 7(b)). After HepG2 cells were treated with Chol-M-Cou and GA-M-Cou, respectively, according to Figure 8, fluorescence microscopy images showed that GA-M-Cou micelles displayed obviously higher coumarin fluorescence in HepG2 cells than the Chol-M-Cou micelles.

These results indicated that GA-M-Cou has a better ability of binding to HepG2 than Chol-M-Cou and the treatment time-dependent GA-M-Cou uptakes behavior of HepG2. These results suggest that the glycyrrhetinic acid fragment of the corresponding block copolymer indeed enhances micelles binding to liver cells.

\subsection{Tissue Distribution of GA-PEG-GA Micelle. As shown} in Figure 9, there were significant differences in tissue distribution between free coumarin and GA-M-Cou in rats. The liver was the only tissue with detectable fluorescence after injection of coumarin loaded GA-PEG-GA micelles intravenous. Administration of the mixture of free coumarin and blank GA-PEG-GA micelles resulted in a nonspecific tissue distribution of fluorescence (Figure 9, upper row), confirming that the hepatic selectivity of GA-PEG-GA micelles was indeed attributed to the GA fragment.

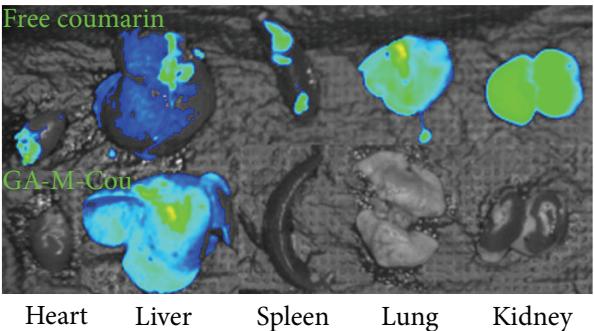

FIGURE 9: The tissue distribution of free coumarin and coumarin loaded GA-PEG-GA micelle.

\section{Conclusions}

In conclusion, the convenient, economic, and effective methods to prepare hepatic targeting polymeric micelles, the surfaces of which are anchored with GA, have been successfully developed for hepatic targeted drug delivery both in vitro and in vivo. Poorly soluble antitumor drug paclitaxel as model is loaded into the micelles. The micelles are spheroids with regular shape and have a size distribution of about $150 \mathrm{~nm}$ and slightly negative surface charge. Flow cytometry 
results and fluorescence spectroscopy images suggest that the presence of glycyrrhetinic acid on the surfaces of the micelles promotes their uptakes by hepatoma cell lines. Thus the paclitaxel loaded GA-PEG-GA micelles have enhanced cytotoxicity of paclitaxel for hepatocellular carcinoma cells, which was confirmed by MTT experiment. Although further investigation on the in vivo antitumor effect of GA-PEG-GA micelles is required, the findings of our study represent an important step in advancing the use of GA-PEG-GA micelles as a potent strategy to treat hepatic carcinoma.

\section{Conflict of Interests}

The authors declare no conflict of interests.

\section{Authors' Contribution}

Fengbo Wu and Ting Xu contributed equally to this work.

\section{Acknowledgments}

The authors apologize to the many research groups whose work could not be cited here due to space limitations. The authors gratefully acknowledge the support by the National Natural Science Foundation of China (nos. 81001357 and 81273471).

\section{References}

[1] M. N. Nassiri Asl and H. Hosseinzadeh, "Review of pharmacological effects of Glycyrrhiza sp. and its bioactive compounds," Phytotherapy Research, vol. 22, no. 6, pp. 709-724, 2008.

[2] J. M. Luk, Q.-S. Zhang, N. P. Lee et al., "Hepatic stellate celltargeted delivery of M6P-HSA-glycyrrhetinic acid attenuates hepatic fibrogenesis in a bile duct ligation rat model," Liver International, vol. 27, no. 4, pp. 548-557, 2007.

[3] S. Shibata, "A drug over the millennia: pharmacognosy, chemistry, and pharmacology of licorice," Yakugaku Zasshi, vol. 120, no. 10, pp. 849-862, 2000.

[4] T. Rossi, M. Castelli, G. Zandomeneghi et al., "Selectivity of action of glycyrrhizin derivatives on the growth of MCF-7 and HEP-2 cells," Anticancer Research, vol. 23, no. 5 A, pp. 3813-3818, 2003.

[5] D. Liu, D. Song, G. Guo et al., "The synthesis of $18 \beta$ glycyrrhetinic acid derivatives which have increased antiproliferative and apoptotic effects in leukemia cells," Bioorganic and Medicinal Chemistry, vol. 15, no. 16, pp. 5432-5439, 2007.

[6] S.-J. Mao, Y.-Q. Bi, J. Hui, D.-P. Wei, H. Ru, and S.-X. Hou, "Preparation, characterization and uptake by primary cultured rat hepatocytes of liposomes surface-modified with glycyrrhetinic acid," Pharmazie, vol. 62, no. 8, pp. 614-619, 2007.

[7] C. Zhang, W. Wang, T. Liu et al., "Doxorubicin-loaded glycyrrhetinic acid-modified alginate nanoparticles for liver tumor chemotherapy," Biomaterials, vol. 33, no. 7, pp. 2187-2196, 2012.

[8] X. H. Wang, Q. Tian, W. Wang, C. N. Zhang, P. Wang, and Z. Yuan, "In vitro evaluation of polymeric micelles based on hydrophobically-modified sulfated chitosan as a carrier of doxorubicin," Journal of Materials Science-Materials in Medicine, vol. 23, no. 7, pp. 1663-1674, 2012.
[9] Q. Tian, X. H. Wang, W. Wang, C. N. Zhang, P. Wang, and Z. Yuan, "Self-assembly and liver targeting of sulfated chitosan nanoparticles functionalized with glycyrrhetinic acid," Nanomedicine-Nanotechnology Biology and Medicine, vol. 8, no. 6, pp. 870-879, 2012.

[10] C. Zhang, Y. Wu, T. Liu et al., "Antitumor activity of drug loaded glycyrrhetinic acid modified alginate nanoparticles on mice bearing orthotopic liver tumor," Journal of Controlled Release, vol. 152, pp. E111-E113, 2011.

[11] Q. Tian, X. Wang, W. Wang, C. Zhang, Z. Yuan, and X. Chen, "Understanding the role of the C3-hydroxyl group in glycyrrhetinic acid on liver targeting," Journal of Controlled Release, vol. 152, pp. E237-E239, 2011.

[12] W. Huang, W. Wang, P. Wang et al., "Glycyrrhetinic acidfunctionalized degradable micelles as liver-targeted drug carrier," Journal of Materials Science Materials in Medicine, vol. 22, no. 4, pp. 853-863, 2011.

[13] W. Huang, P. Wang, W. Wang et al., "Preparation of glycyrrhetinic acid-modified PEG-PLGA nanoparticles and the affinity evaluation on hepatoma cells," Chemical Journal of Chinese Universities, vol. 32, no. 2, pp. 416-420, 2011.

[14] W. Huang, W. Wang, P. Wang et al., "Glycyrrhetinic acidmodified poly(ethylene glycol)-b-poly( $\gamma$-benzyl l-glutamate) micelles for liver targeting therapy," Acta Biomaterialia, vol. 6, no. 10, pp. 3927-3935, 2010.

[15] C. Zhang, W. Wang, C. Wang et al., "Cytotoxicity of liver targeted drug-loaded alginate nanoparticles," Science in China: Series B, vol. 52, no. 9, pp. 1382-1387, 2009.

[16] Q. Tian, W. Wang, X. He et al., "Glycyrrhetinic acid-modified nanoparticles for drug delivery: preparation and characterization," Chinese Science Bulletin, vol. 54, no. 18, pp. 3121-3126, 2009.

[17] R.-T. Zha, X.-T. He, T. Du, and Z. Yuan, "Synthesis and characterization of chitosan nanoparticles modified by glycyrrhetinic acid as a liver targeting drug carrier," Chemical Journal of Chinese Universities, vol. 28, no. 6, pp. 1098-1100, 2007.

[18] Q. Tian, C.-N. Zhang, X.-H. Wang et al., "Glycyrrhetinic acidmodified chitosan/poly(ethylene glycol) nanoparticles for livertargeted delivery," Biomaterials, vol. 31, no. 17, pp. 4748-4756, 2010.

[19] Z. Y. He, X. Zheng, X. H. Wu et al., "Development of glycyrrhetinic acid-modified stealth cationic liposomes for gene delivery," International Journal of Pharmaceutics, vol. 397, no. 12, pp. 147-154, 2010.

[20] H. Inoue, T. Mori, S. Shibata, and Y. Koshihara, "Modulation by glycyrrhetinic acid derivatives of TPA-induced mouse ear oedema," British Journal of Pharmacology, vol. 96, no. 1, pp. 204210, 1989.

[21] S.-J. Um, M.-S. Park, S.-H. Park, H.-S. Han, Y.-J. Kwon, and H.S. Sin, "Synthesis of new glycyrrhetinic acid (GA) derivatives and their effects on tyrosinase activity," Bioorganic and Medicinal Chemistry, vol. 11, no. 24, pp. 5345-5352, 2003.

[22] H. Takahashi, H. Onishi, and Y. Machida, "Glycyrrhetic acidloaded microparticles: liver-specific delivery and therapeutic potential against carbon tetrachloride-induced hepatitis," Journal of Pharmacy and Pharmacology, vol. 56, no. 4, pp. 437-444, 2004.

[23] C. Puglia, C. Ostacolo, A. Sacchi, S. Laneri, and F. Bonina, "Invitro and in-vivo evaluation of oligoethylene esters as dermal prodrugs of $18 \beta$-glycyrrhetic acid," Journal of Pharmacy and Pharmacology, vol. 58, no. 3, pp. 311-319, 2006. 
[24] G. He, Z. He, X. Zheng et al., "Synthesis, characterization and in vitro evaluation of self-assembled poly(ethylene glycol)glycyrrhetinic acid conjugates," Letters in Organic Chemistry, vol. 9, no. 3, pp. 202-210, 2012.

[25] Q. Tian, X. Wang, W. Wang, C. Zhang, Y. Liu, and Z. Yuan, "Insight into glycyrrhetinic acid: the role of the hydroxyl group on liver targeting," International Journal of Pharmaceutics, vol. 400, no. 1-2, pp. 153-157, 2010.

[26] H. Li, Y. Shao, X. Wang, S. Quan, Y. Zhang, and Z. Zhang, "Oligodeoxynucleotide acid loaded, NGR-peptide-conjugated polymeric liposomes: in vitro and in vivo evaluation," Current Nanoscience, vol. 7, no. 5, pp. 797-806, 2011.

[27] M. Ramezani, B. Malaekeh-Nikouei, S. Malekzadeh, M. R. Baghayeripour, and M. Malaekeh-Nikouei, "The effect of lipopolymer structure on the transfection efficiency of hydrophobic polyethylenimine-based cationic nanoliposomes," Current Nanoscience, vol. 8, no. 5, pp. 680-684, 2012.

[28] J. Li, Z. He, S. Yu et al., "Micelles based on methoxy poly(ethylene glycol)-cholesterol conjugate for controlled and targeted drug delivery of a poorly water soluble drug," Journal of Biomedical Nanotechnology, vol. 8, no. 5, pp. 809-817, 2012.

[29] L. Cai, N. Qiu, X. Li et al., "A novel truncated basic fibroblast growth factor fragment-conjugated poly (ethylene glycol)cholesterol amphiphilic polymeric drug delivery system for targeting to the FGFR-overexpressing tumor cells," International Journal of Pharmaceutics, vol. 408, no. 1-2, pp. 173-182, 2011. 

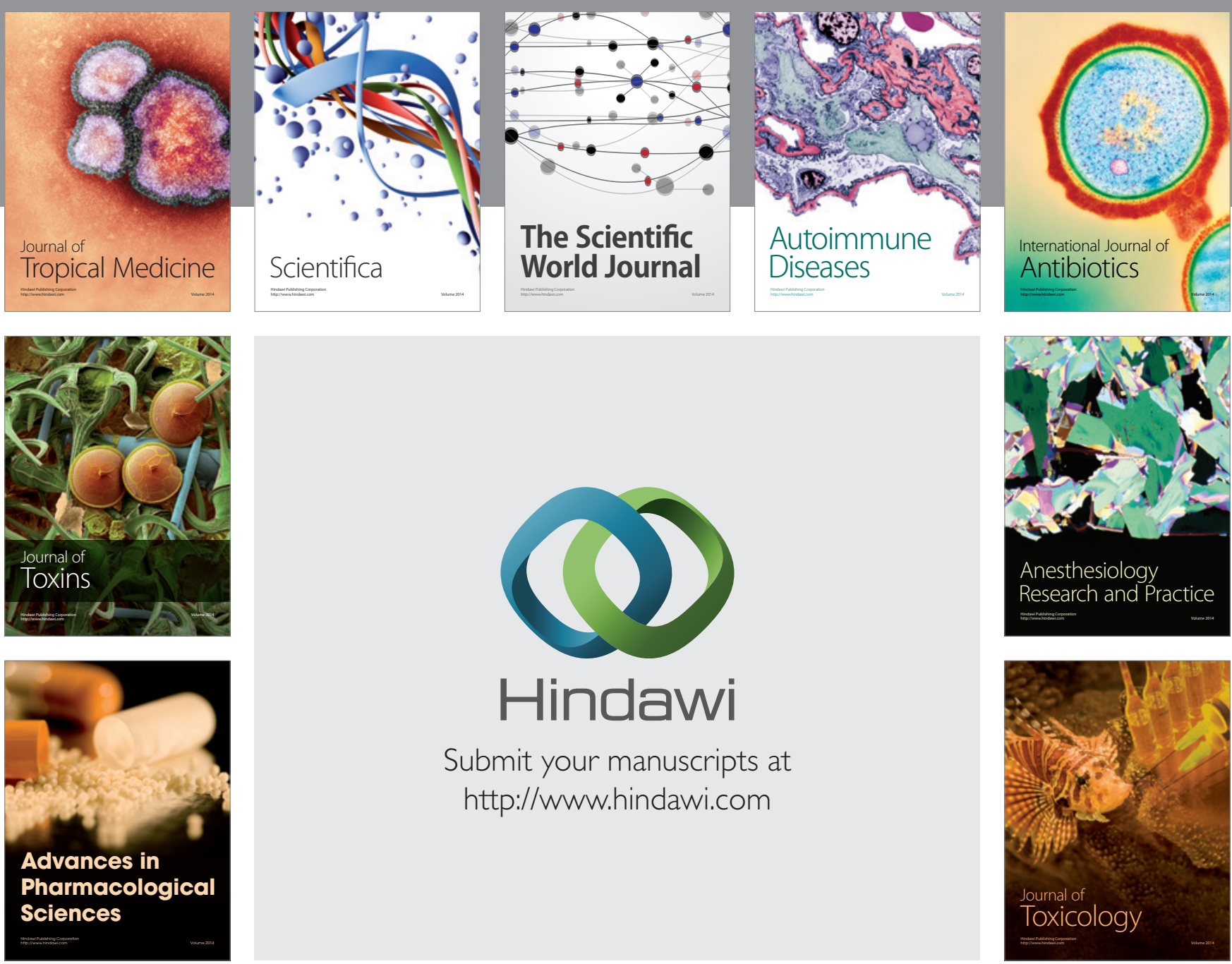

\section{Hindawi}

Submit your manuscripts at

http://www.hindawi.com
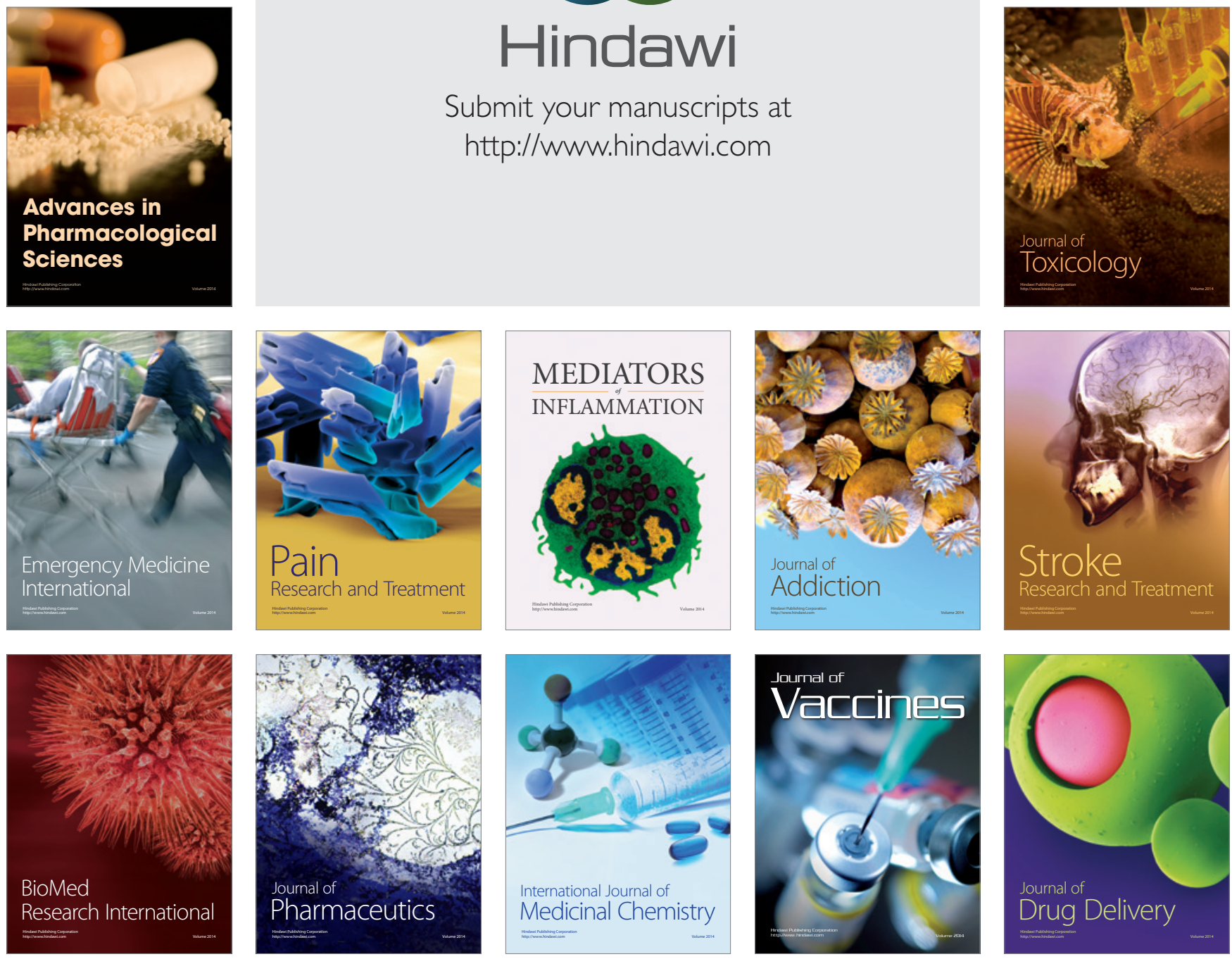